\title{
DESIGN OPTIMIZATION OF ROTOR PROPULSIVE DELTA WING
}

\section{THILAKRAJ, V. RAVI, \& K. SUNIL CHARKRAVARTHY}

Malla Reddy College of Engineering and Technology, Hyderabad, India

\section{ABSTRACT}

The Wing Rotor Configuration is designed for the purpose of VTOL (vertical takeoff and landing) which is a combination of Delta wing and rotor together integrated at the empennage section of the aircraft wing. The model of the delta wing rotor configuration will be modelled by using the solid works part modelling software, later the model will be studied in cfd to understand the aerodynamic performance of the aircraft. The objective of the work is to achieve high aerodynamic efficiency of the chosen configuration. The model analysis includes static and CFD analysis where flow analysis is the primary function, deflection and stress distribution is calculated in the static analysis in ansys static structural module and velocity streamlines and pressure distribution is calculated in the flow analysis which is done using the CFD module of ansys software, major applications include VTOL of different aircrafts [majorly UAV'S (unmanned aerial vehicle), MAV'S (micro aerial vehicle), and fighter aircrafts. ${ }^{[1]}$

KEYWORDS: VTOL (Vertical Takeoff and Landing), CFD, MAV'S (Micro Aerial Vehicle) \& Fighter Aircrafts

Received: May 27, 2019; Accepted: Jun 17, 2019; Published: Jul 13, 2019; Paper Id.: IJMPERDAUG201991

\section{INTRODUCTION}

The Vertical Take-Off and Landing (VTOL) aircraft are able to take-off with the agility of a helicopter while retaining the efficiency and speed of an airplane during conventional flight. The applications of such an aircraft are vast, ranging from civilian transport to aero medical evacuation and troop deployment. Nowadays these aircraft have been limited to military and research applications. The long range and efficiency associated with conventional aircraft means they can cover large distances and the can land where conventional aircraft often cannot. In these locations supplies can be deployed or surveillance obtained without the need for a sizeable landing area. The wing rotor configuration provides vertical takeoff and landing with the help of delta wing and rotor combination, there are multiple vtol aircrafts available and each of them have their own specific advantage. ${ }^{[4]}$

There are two types of vtol crafts available till now they are:

- Helicopters and

- $\quad$ Tilt engines.

This delta wing rotor design is the best possible model, which can be used in various fields to meet its needs. This vtol crafts doesn't need any runway as they can take off and land vertically with help of rotors.

This model is very much suitable in real time applications. The model development done using solid works 2018 and analysis in done ansys workbench 2018. The analysis is done in both static structural module and computational fluid dynamics CFD module. 


\section{AIM}

Even though there are multiple types of vtol purpose vessels are available, the main objective of this work is to find out the best suitable model for the vtol application provided with deltawing and rotor combination. The delta wing rotor model is the unique model which serves the vtol purpose here the analysis of the model was done and the output is the behavior of the model to various speed inputs and different material as input given thus the suitability of the model in real time applications can be found. ${ }^{[2]}$

The aim of this work is to design and analyze the delta wing rotor configuration and find the aerodynamic efficiency along with stability of the model under the varying loads.

\section{MODEL PREPARATION}

The preparation of the model using solid works, after entering the sketcher module and selecting front plane for the 2D drawing, and using the point command NACA0012 profile is drawn using $\mathrm{x}$ and y coordinates then using spline command the points are joined to create a closed profile of airfoil later using convert entities scaling of the profile is done to100 times to get desired size, then by taking a reference plane at distance of $400 \mathrm{~mm}$ and projection of the the sketch on to the plane is done and scaling is done again to 4 times to get the delta wing component. ${ }^{[3]}$

The following pictures show the $2 \mathrm{~d}$ geometry of naca0012 profile and the projected scaled profile.

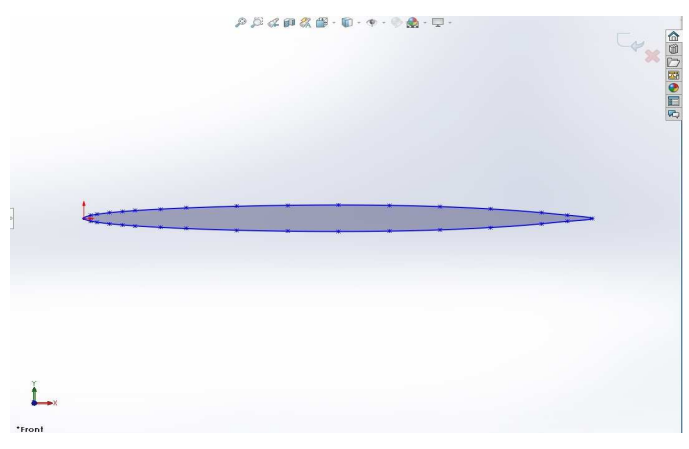

Figure 1: Aerofoil 2d Geometry

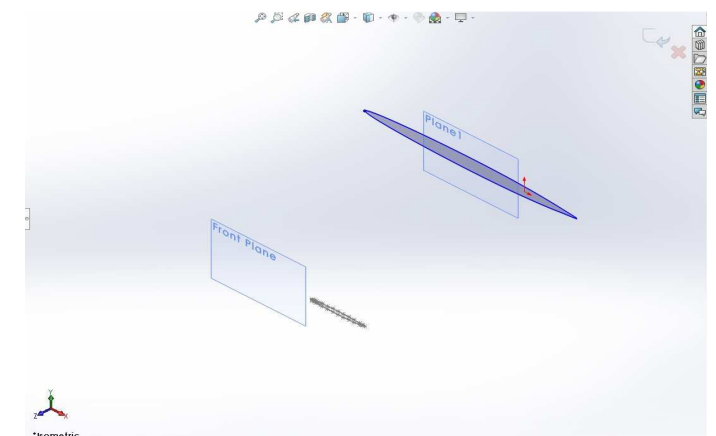

Figure 2: Aerofoil 2d Geometry Scaled Model

Then exit the sketcher module and using the loft command joining of the two profiles is done to create a solid $3 \mathrm{~d}$ model of the delta wing later by creating a circle on the top plane at the aerodynamic centre of the wing and then exiting the sketcher module, using extruded cut command removal of the material inside the circle is done to create a wing component with hole for the rotor placement purpose.

Numerical optimization technique used for the placement of the rotor on the wing at the suitable position thus the geometry should be more efficient and effective for the desired purpose.

Using the optimization technique, the suitable positions for rotor are, from the top view of wing geometry (reference point as smaller end of wing's leading edge) :

$$
\begin{aligned}
& X=-37.50, Y=250 \\
& X=-37.50, Y=265 \\
& X=-37.50, Y=275
\end{aligned}
$$




$$
\begin{aligned}
& X=-31.25, Y=265 \\
& X=-31.25, Y=275 \\
& X=-43.75, Y=265 \\
& X=-43.75, Y=250 \\
& X=-25.00, Y=250
\end{aligned}
$$

Among these points the rotor found to be more efficient in the $\mathrm{X}=-37.50, \mathrm{Y}=265$ coordinates as per observation.

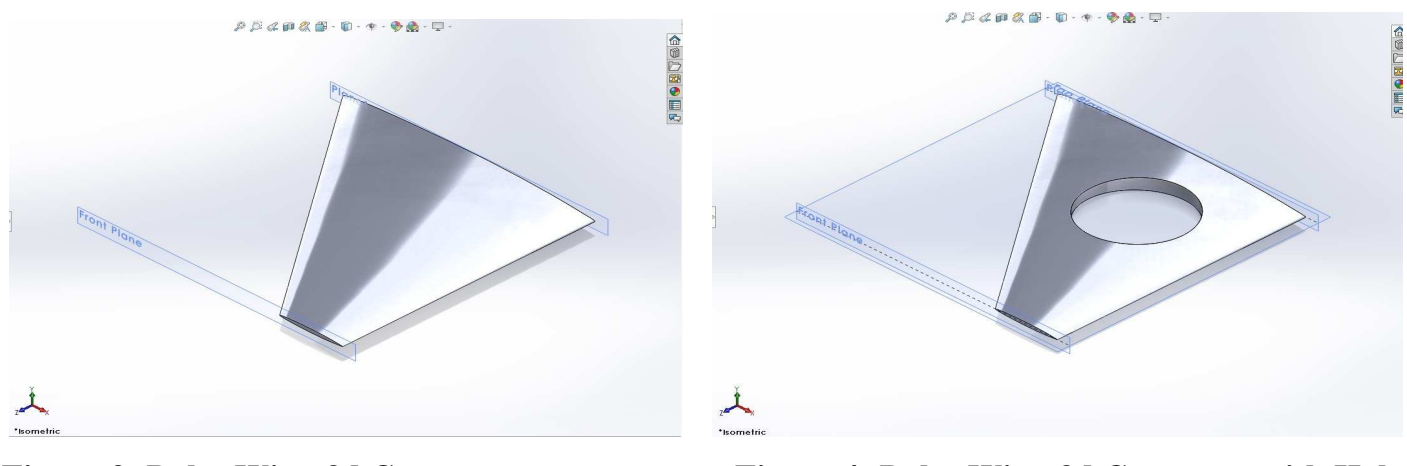

Figure 3: Delta Wing 3d Geometry

Figure 4: Delta Wing 3d Geometry with Hole

After finishing the wing geometry the second component should be a support for rotor to create this component first by taking a cylindrical component in the middle using top plane and we also create another cylinder on top of the support for fixing the rotor and by taking front plane as reference a plane is created at such distance so that the radius from cylinder to the plane should be equal to that of the hole of wing geometry.

Then on the new plane a straight slot is created and extruded upto the cylindrical surface then by taking circular pattern of the solid slot creation of the support is done later the developed support geometry was cut from the top plane to match the hole of wing geometry.

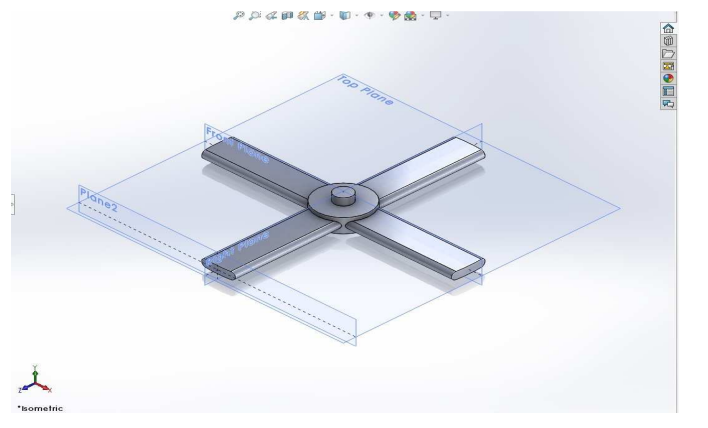

Figure 5: Rotor Support

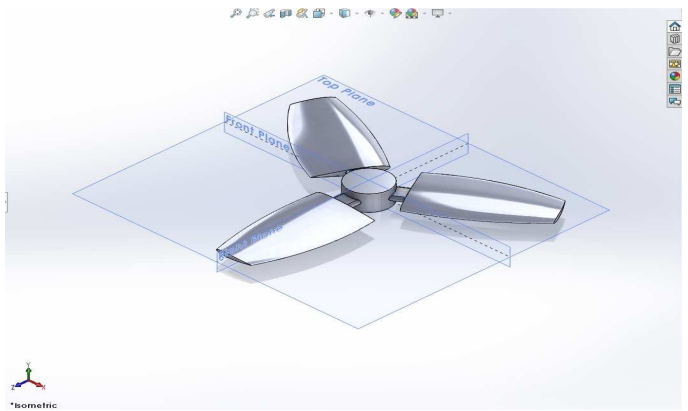

Figure 6: Rotor

After the creation of the wing geometry and the support final component is creating the rotating fan or rotor for this first the cylindrical component was created as support for blades similar to the previous one on bottom of this the small cylindrical cut was made so that the rotor will be placed on top of the support.

A reference plane was created from front plane and the naca0016 profile was created exactly on the middle of the plane and by taking the front and reference plane another plane was created at the middle and the sketch is projected onto the plane and scaled to double the size the same sketch was projected onto the front plane later by using these three profile 
using loft command solid wing rotor was generated, and then the slot was created on it to attach it to the cylinder and the blade and slot are tripled using the circular pattern command. The final rotor was then created by removing the excess material from top surface with $140 \mathrm{~mm}$ diameter.

After creating the three components, they are assembled in the assembly module, as shown in the above figure the components are joined using mate command. The support and wing are joined and fixed together as a single component where the rotor is placed over the support is allowed to rotate.

Horizontal and vertical distance of the wing geometry is $400 \mathrm{~mm}$ and the hole is $150 \mathrm{~mm}$ diameter, rotor diameter is $140 \mathrm{~mm}$ and the support having equal diameter of the wing hole geometry i. e., $150 \mathrm{~mm}$.

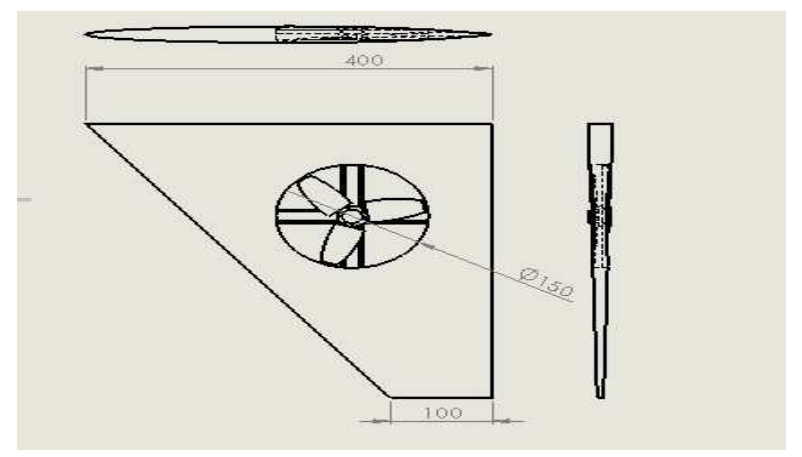

Figure 7: Deltawing Rotor Model [All Dimensions are in Mm]

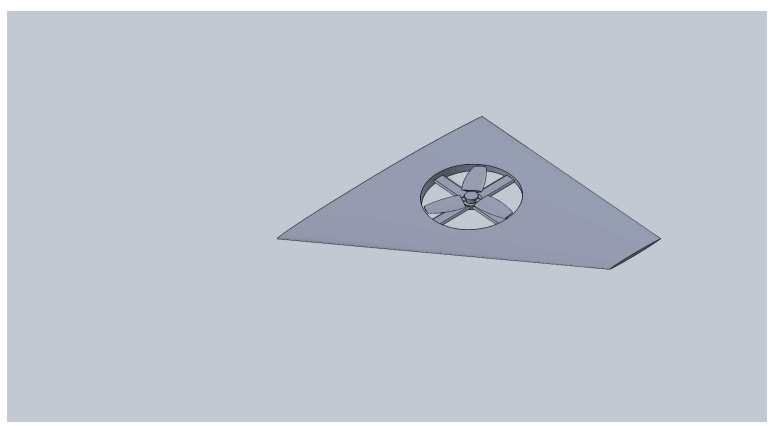

Figure 8: Deltawing Rotor Configuration [Isometric View]

\section{MODEL ANALYSIS}

The model analysis done using ansys workbench the model was imported into the static structural module and then in the engineering data section the data was provided for the titanium aluminium alloy. ${ }^{[3]}$

\section{BOUNDARY CONDITIONS:}

Young's Modulus: $110 \mathrm{GPa}$

Poisson's Ratio: 0.33

Density: $4725 \mathrm{~kg} / \mathrm{m} 3$

Load applied: $500 \mathrm{KPa}$

The geometry module is used to generate the model and using Boolean operations command the wing and support are united later the module was closed and the mesh module was opened and the model is meshed. 


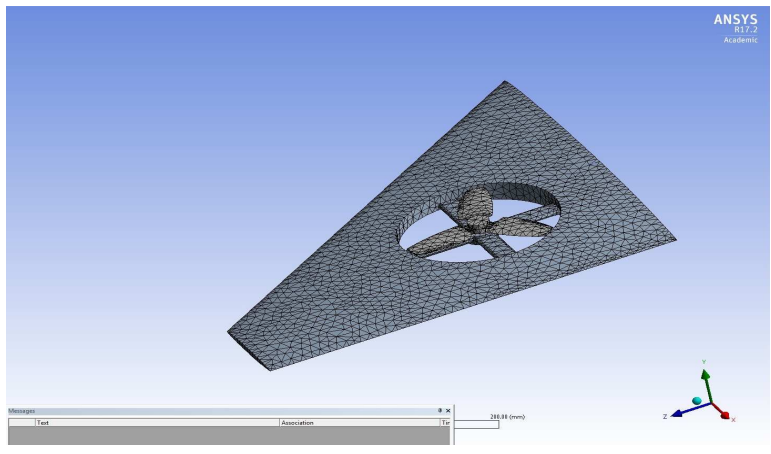

Figure 9: Meshed Model

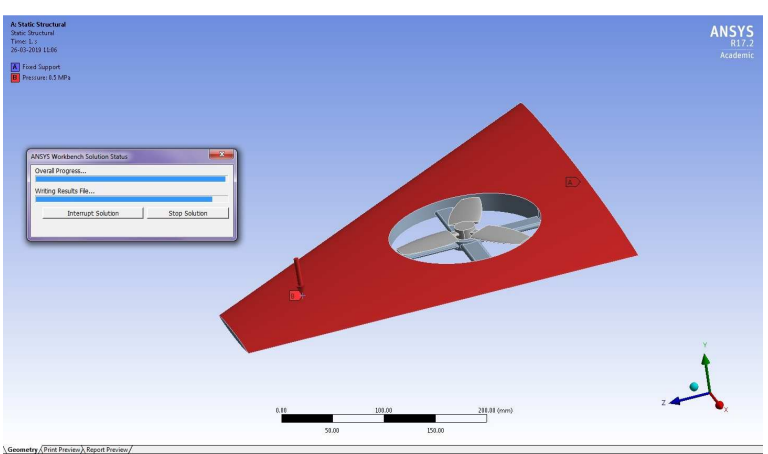

Figure 10: Pressure Applied on Model

After the mesh the model was opened in the setup module the bigger end of the wing is fixed and the pressure is applied over the top surface with about 500kpa. Then the problem was solved using calculations command and the total stress, deformation and maximum principle stress is found as shown in the above figures.

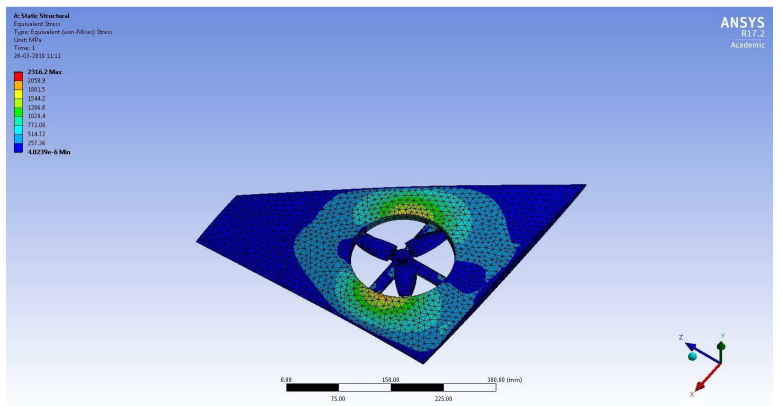

Figure 11: Equivalent Stress

Maximum and minimum equivalent stress values are $2316.2 \mathrm{Mpa}$ and $4.0239 \mathrm{e}-6 \mathrm{Mpa}$ and the average value is 1286.8 Mpa.

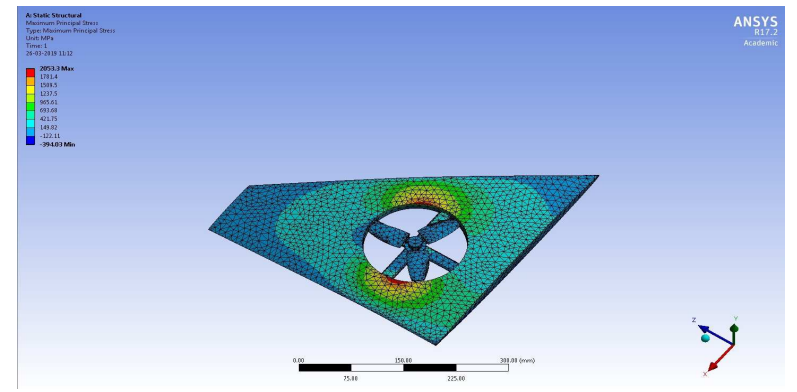

Figure 12: Maximum Principle Stress 
Maximum and minimum principle stress values are 2053.3Mpa and $-394.03 \mathrm{Mpa}$ and the average value is 965.61 Mpa.

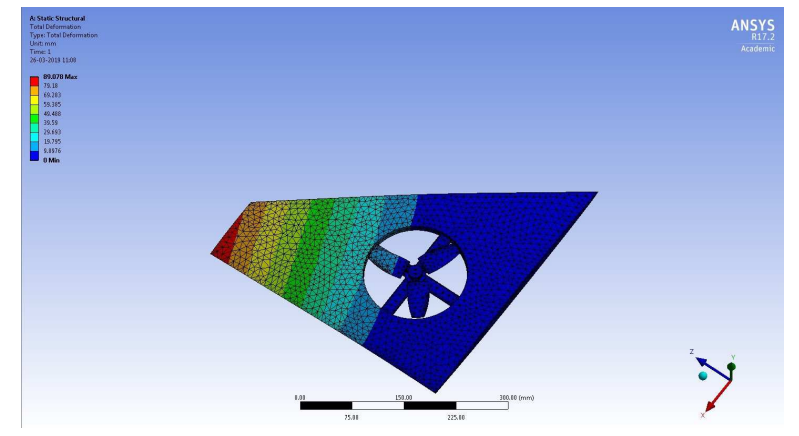

Figure 13: Total Deformation

Maximum and minimum total deformation values are $89.078 \mathrm{~mm}$ and $0 \mathrm{~mm}$ and the average value is $49.489 \mathrm{~mm}$. the deflection is maximum at the tip and minimum at the fixed end.

From the above results the deflection and stress distribution were found to be satisfying compared to the previous research works. ${ }^{[3]}$

\section{FLOW ANALYSIS}

The analysis begins with the model being imported into ansys work bench cfd module then the model is generated in the geometry section, later using Boolean operations the model wing and support are united into a single component then an enclosure is created about $3000 \mathrm{~mm}^{3}$ and the model is placed at the middle of it i. e, is at $-1500 \mathrm{~mm}$ then using Boolean operation the model was subtracted from the enclosure then geometry module was closed. ${ }^{[6]}$

Later the mesh module was opened and using design module the model was meshed, then according to requirement relevance was changed and the front face was selected, and by using the named selection command the name was changed to inlet. Similarly the opposite face is selected and the named selection was changed as outlet and all other faces was selected and named as walls and the mesh module was closed.

Then using the setup module the boundary is created and named as inlet and the velocity is added as $30 \mathrm{~m} / \mathrm{s}$ and the pressure is maintained as 101325 pa or 1.01 bar and another boundary is created as outlet with the relative pressure as 0 and another one with walls apply and select the air as ideal gas then close the set up.

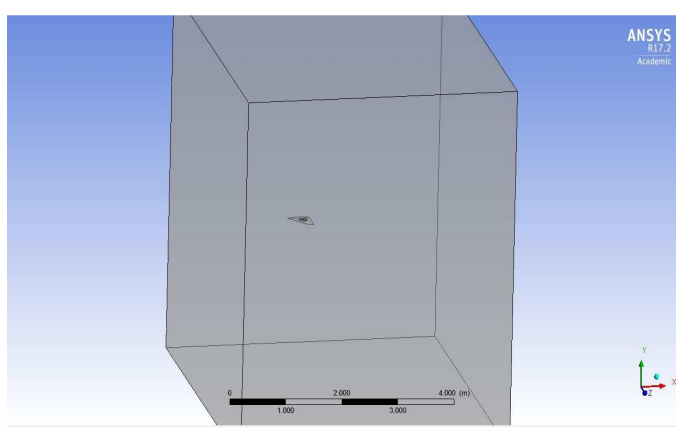

Figure 14: Creation of an Enclosure

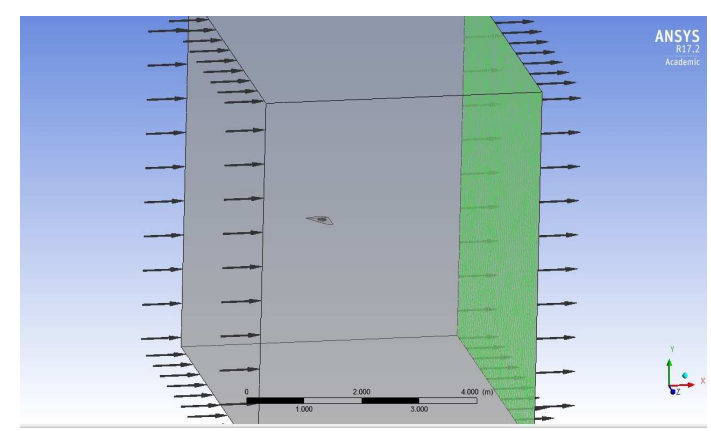

Figure 15: Applying Inlet Velocity

The solution command is selected and in the results module create a velocity stream line, then at the inlet add 3000 points apply and create a plane using user location insert plane later on the plane create velocity stream line points as 
3000 and apply create another plane xz then insert the stream line at plane 2 with 3000 points, create a contour as pressure on plane apply.

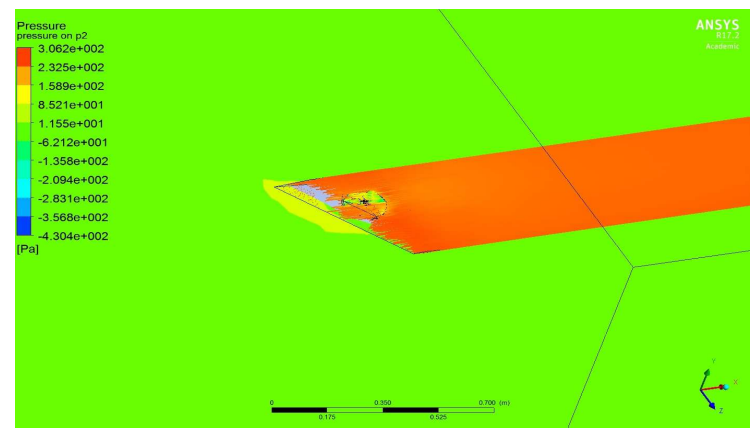

Figure 16: Pressure on Plane2

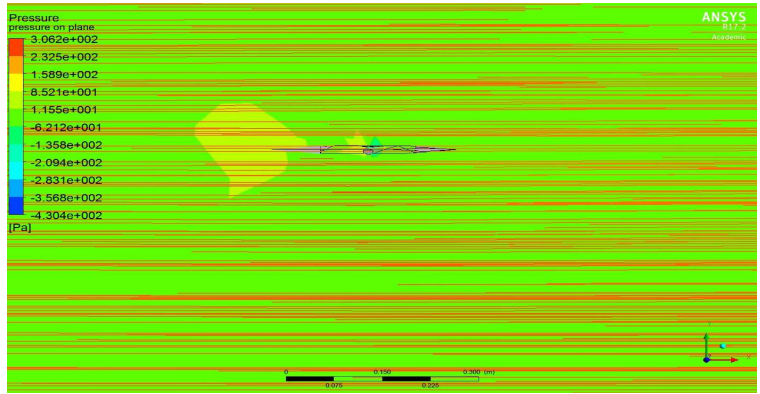

Figure 17: Pressure on Plane1

Maximum and minimum pressure values on plane 2 are $3.062 \mathrm{e}+002 \mathrm{pa}$ and $-4.304 \mathrm{e}+002 \mathrm{pa}$ and the average value is $1.155 \mathrm{e}+001 \mathrm{pa}$.

It's observed that the pressure around the leading edge is found to be $1.58 \mathrm{e}+002 \mathrm{pa}$, and the pressure around the rotor is found to be fluctuating from $2.325 \mathrm{e}+002 \mathrm{pa}$ to $1.155 \mathrm{e}+001 \mathrm{pa}$.

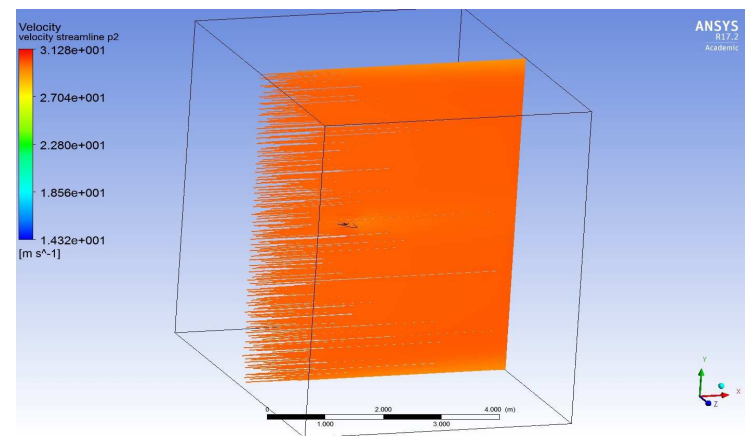

Figure 18: Velocity Streamline on Plane2 


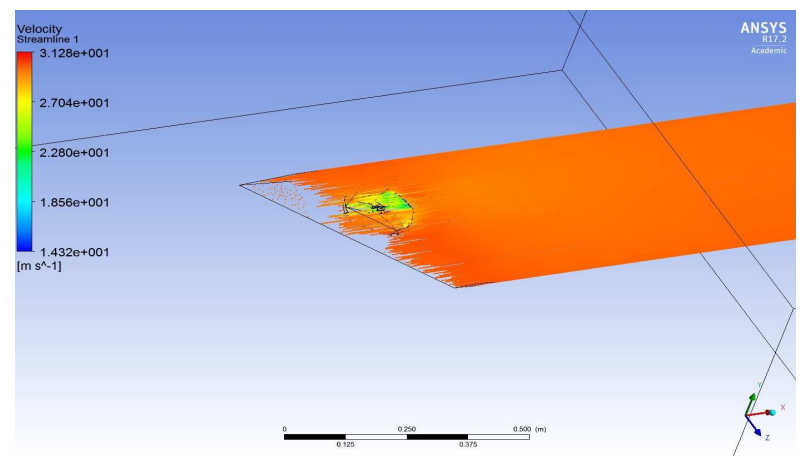

Figure 19: Velocity Streamline on Plane1

Maximum and minimum velocity streamline values on plane 2 and 1 are $3.128 \mathrm{e}+001 \mathrm{e}+001 \mathrm{~ms}^{\wedge}-1$ and $1.432 \mathrm{e}+001 \mathrm{~ms}^{\wedge}-1$ and the average value is $2.280 \mathrm{e}+001 \mathrm{~ms}^{\wedge}-1$.

\section{RESULTS AND CONCLUSION}

The static analysis of the model shows the deformation and stress distribution are in acceptable range. According to the analytical report, the component is in much good position to uphold the pressure and maintain its strength and much suitable for subsonic flights but still further research is going and that will explains the prefect feature of this upcoming configuration, but for now it is feasible in nature.

The behavior of model in the subsonic speeds is widely acceptable in nature and because of the rotor fixture the model cannot achieve higher speeds even though the model is good in subsonic region when the speed is increased to sonic and above the rotor geometry is taking heavy loads and creating shocks thus by increasing drag.

The very purpose of the design is to operate the model with vtol and for the rescue, emergency, and military operation and these applications are of limited range only hence subsonic speeds are acceptable and the delta wing rotor configuration model is suitable for these applications.

\section{FUTURE SCOPE}

The deltawing rotor model is suitable for multiple applications like search and rescue operations, military missions, emergency response systems etc., all these application need a vtol carrier because runways might not be available in remote areas. Since there is large scope to modify this model multiple times it is possible to tilt the rotor to 90 degrees and use it as prime mover.

In future, this technology can be adaptable for daily use by importing this method to create flying cars. The wing rotor configuration can be placed at the side door of the cars thus can be used similar to a quad copter.

This wing rotor configuration is best suitable in UAV (unmanned aerial vehicles) and MAV (manned aerial vehicle).

\section{REFERENCES}

1. VTOL-WIKIPEDIA: http://en.wikipedia.org/wiki/VTOL

2. VTOL Aircraft by J F Coplin, published by Macdonald \& Co (Publishers) Ltd, London, 1967.

3. International Journal of Scientific \& Engineering Research, Volume 6, Issue 4, April-2015 461 ISSN 2229-5518 
4. International Journal of Innovative Research in Advanced Engineering (IJIRAE), ISSN: 2349-2163 Issue 2, Volume 2 (February 2015)

5. Anderson, John D., Jr., Fundamentals of Aerodynamics, $3^{\text {rd }}$ edition, McGraw-Hill Book Company, New York,1984.

6. Sivakumar, S., Ranjithkumar, N., \& Ragunathan, S. (2013). Design and development of down draft wood gasifier. International Journal of Mechanical Engineering, 2(2), 1-10.

7. P. k. Kundu, I. M. cohen. Fluid mechanics 3/e. Academic press, Indian Reprint 2005.

8. HUNECKE K. 'Jet Engines: Fundamentals of theory, Design and Operation', England. 1997. 
